PROCEEDINGS OF THE

AMERICAN MATHEMATICAL SOCIETY

Volume 133, Number 6, Pages 1843-1850

S 0002-9939(05)07742-7

Article electronically published on January 21, 2005

\title{
STEIN FILLABILITY AND THE REALIZATION OF CONTACT MANIFOLDS
}

\author{
C. DENSON HILL AND MAURO NACINOVICH
}

(Communicated by Jon G. Wolfson)

\begin{abstract}
There is an intrinsic notion of what it means for a contact manifold to be the smooth boundary of a Stein manifold. The same concept has another more extrinsic formulation, which is often used as a convenient working hypothesis. We give a simple proof that the two are equivalent. Moreover it is shown that, even though a border always exists, its germ is not unique; nevertheless the germ of the Dolbeault cohomology of any border is unique. We also point out that any Stein fillable compact contact 3-manifold has a geometric realization in $\mathbb{C}^{4}$ via an embedding, or in $\mathbb{C}^{3}$ via an immersion.
\end{abstract}

Let $M$ be a smooth orientable compact real $(2 n+1)$-dimensional manifold without boundary $(n=1,2,3, \ldots)$. Let $\Xi$ be a smooth orientable contact structure on $M$. The orientation of $\Xi$ is defined by a global contact form $\xi$ on $M$, with $\Xi=\{v \in T X \mid \xi(v)=0\}$, and which is strongly non-integrable, so that $\omega=\xi \wedge(d \xi)^{n}$ is $\neq 0$ at each $x \in M$, so defining an orientation of $M$. We shall always take $\omega$ as the orientation of $M$, and we shall say then that $M$ and $\Xi$ are equally oriented.

Assume that the contact manifold $(M, \Xi)$ is the smooth boundary of a Stein manifold $(X, J)$.

Let us clarify this notion: Let $X$ be a complex manifold, of dimension $(n+1)$, with a smooth boundary $M$. Assuming that its complex structure $J$ is smooth up to the boundary $M$, it induces a smooth $C R$ structure $\left(M, H M, J_{M}\right), J_{M}: H M \rightarrow H M$, $J_{M}^{2}=-I$ of hypersurface type $(n, 1)$ on $M$. To say that a contact structure $\Xi$ on $M$ is induced by the $C R$ structure of $M$ means that $\Xi=H M$ are the same distribution of $2 n$-planes in $T M$. Since $M$ is a boundary, the contact structure $\Xi$ is orientable and a global contact form $\xi$ defines the Levi form of $M$ :

$$
H M \ni v \rightarrow d \xi\left(J_{M} v, v\right) \in \mathbb{R} .
$$

This is a Hermitian form on $H M$ for the complex structure $J_{M}$. The strong nonintegrability condition $\xi \wedge(d \xi)^{n} \neq 0$, together with the formal integrability of the partial complex structure $J_{M}$, imply that for each $x \in M$ the Levi form $H_{x} M \ni$ $v \rightarrow \mathcal{L}(v) \in \mathbb{R}$ is non-degenerate, i.e. all its eigenvalues are different from zero.

In particular, when $M$ is the boundary of a Stein manifold $X$, the Levi form $\mathcal{L}$ of $M$ is positive definite at every $x \in M$ : in this case the induced $C R$ structure is strongly pseudoconvex. In this situation it is customary to say that "the contact manifold $M$ is Stein fillable by $X "$.

Received by the editors November 19, 2003 and, in revised form, March 2, 2004.

2000 Mathematics Subject Classification. Primary 53D10, 32V15, 35N99.

Key words and phrases. Stein manifold, contact manifold.

(c) 2005 American Mathematical Society Reverts to public domain 28 years from publication 1843 
The purpose of this note is to delve into the issue of the meaning of the sentence in italics.

\section{ThE INTRINSIC NOTION}

Here is the issue: What is meant by saying that $M$ is the smooth boundary of a complex manifold $X$ ? If we are to enjoy the convenience and flexibility of a differential topologist, and want to work in the smooth $\left(\mathcal{C}^{\infty}\right)$ category, then the intrinsic notion is clear. It goes as follows:

(i) $\bar{X}=X \cup M$ has the structure of a $\mathcal{C}^{\infty}$ manifold with a $\mathcal{C}^{\infty}$ boundary $M$, $X$ being the interior of $\bar{X}$.

(ii) $X$ is endowed with a formally integrable almost complex structure $J$ : $T X \rightarrow T X, J^{2}=-I$, which is $\mathcal{C}^{\infty}$ up to the boundary $M$.

[This much gives us a smooth induced almost- $C R$ structure $J_{M}$ on $M$, which in turn induces a distribution of $2 n$-planes $\Xi=H M$ on $M$. When $n=1$, there are no integrability conditions and in fact the $C R$ structure can be taken strictly pseudoconvex if the corresponding contact structure is strongly non-integrable.] For Stein fillability we require in addition that

(iii) $X$ is a Stein manifold.

Remark 1.1. It follows from (ii) via the Newlander-Nirenberg theorem that $X$ has an atlas of interior holomorphic coordinate charts. But it does not follow immediately from the above definition that $\bar{X}$ has an atlas of holomorphic coordinate charts [which would have to include boundary charts]. Nor does it immediately follow from the definition that $\bar{X}$ can be regarded as the closure of a domain in some larger open complex manifold $\widetilde{X}$. See for example the discussion in [H1], [H2], [H3].

\section{A WORKING HYPOTHESIS}

There has been considerable recent interest in compact contact manifolds which are Stein fillable, and many very interesting and significant results have been obtained, especially when $\operatorname{dim}_{\mathbb{R}} M=3$ (see e.g. [El1], [El2], [El3], [Go], [LiM]).

In these articles, however, the intrinsic notion is not always being used; what is being used instead is the following convenient working hypothesis:

$1^{o}$ The Stein manifold $X$ is an open set in a larger open complex manifold $Y$, with $X \Subset Y$.

$2^{o}$ There exists a real $\mathcal{C}^{\infty}$ strictly plurisubharmonic function $\phi$ on $Y$.

$3^{o} \bar{X}=X \cup M=\{x \in Y \mid \phi(x) \leq 0\}$ with $\left.d \phi\right|_{M} \neq 0$.

$4^{o} \phi$ is a Morse function on $Y$; i.e. $\phi$ has at most a finite number of critical points, all of which are nondegenerate.

This working hypothesis clearly implies the intrinsic notion, but it also involves a number of extrinsic elements. In $\S 6$ we give a simple proof that the intrinsic notion is equivalent to the convenient working hypothesis.

\section{Existence AND NON-Uniqueness of the Border}

In this section we do not need that $M$ be compact, nor that $X$ be Stein. But we will tacitly assume that all the manifolds are paracompact (i.e. countable at 
infinity). Otherwise we place ourselves in the position of $(i)$ and $(i i)$ of the intrinsic notion.

Theorem 3.1. Assume that the contact manifold $M$ is the $\mathcal{C}^{\infty}$ intrinsic boundary of a strictly pseudoconvex complex manifold $X$. Then:

(a) $\bar{X}$ is a domain $\bar{X} \subset \widetilde{X}$, having interior $X$ and $\mathcal{C}^{\infty}$ strictly pseudoconvex boundary $M$, in some open complex manifold $\tilde{X}$.

(b) Even though a border $\widetilde{X} \backslash \bar{X}$ exists by (a), its germ along $M$ is, in general, not unique.

Proof. (a) Since by $(i) \bar{X}$ is a smooth manifold with a smooth boundary, there is a $\mathcal{C}^{\infty}$ collar, so that we can consider $\bar{X}$ as a domain in some open real $(2 n+2)$ dimensional smooth manifold $\Omega$. By $(i i)$ there is a complex structure tensor $J$ on $X$ which is $\mathcal{C}^{\infty}$ up to $M$, and hence induces the strictly pseudoconvex structure $J_{M}$ on $M$. Since $J$ is assumed in $(i i)$ to be $\mathcal{C}^{\infty}$ up to the boundary, we may consider its smooth extension $\bar{J}$ to $\bar{X}$, so $J_{M}=\left.\bar{J}\right|_{H M}=\left.\bar{J}\right|_{\Xi}$. Since Whitney sections over closed sets can be continued to smooth sections over open neighborhoods, we may, after possibly shrinking $\Omega$, extend $\bar{J}$ to a smooth almost complex structure $J_{\Omega}$ on $\Omega$, such that $\left.J_{\Omega}\right|_{\bar{X}}=\bar{J}$ satisfies the formal integrability conditions of the Newlander-Nirenberg theorem on $\bar{X} \subset \Omega$. Now the statement (a) is the content of Theorem 1 in HN1], where a detailed proof is given. It tells us that there is an open submanifold $\widetilde{X}$, with $\bar{X} \subset \widetilde{X} \subset \Omega$, and a complex structure $\widetilde{J}$ on $\widetilde{X}$, such that $\left.\widetilde{J}\right|_{\bar{X}}=\bar{J}$. The proof of that theorem involves a tricky use of Zorn's lemma, and employs an up-to-the-boundary version of the Newlander-Nirenberg theorem, which is valid here since $M$ is strictly pseudoconvex (see [HJ], $\mathrm{Ca}]$ ).

This completes the proof of (a).

Remark 3.1. When $M$ is compact, weakly pseudoconvex and of finite type in the sense of D'Angelo (see [DA]), the existence of $\tilde{X}$ was shown by [Ch] using a much more complicated argument. When $M$ is compact, strictly pseudoconvex, and is a boundary in the concrete sense (see [H1]), the existence of $\tilde{X}$ was shown by and $[\mathrm{He}$. Additional very interesting related results were obtained in [Le1], Le2], [Le3.

(b) We give a simple counterexample to uniqueness of the germ of the border along $M$, even in the simple case where $X=B$ is an open ball in $\mathbb{C}^{m}(m=$ $1,2,3, \ldots)$ with boundary $\partial B=S^{2 m-1}$. For convenience take $B$ to be the ball of radius $\frac{1}{2}$ centered at the point $\frac{1}{2} e_{1}$, where $e_{1}=(1,0, \ldots, 0)$. Let $D$ denote the open unit disc in $\mathbb{C}$, and $\omega$ denote a suitable open neighborhood of $\bar{D}$, to be chosen later. We set $U=\omega \times D^{m-1}$ and note that $U$ is an open neighborhood of $\bar{B}$ in $\mathbb{C}^{m}$. On $U$ we have the standard complex structure, which can be described by a single global holomorphic coordinate patch $\left(U ; z_{1}, \ldots, z_{m}\right)$. We shall construct another complex structure on $U$, also described by a single global holomorphic coordinate patch of the form $\left(U ; \widetilde{\phi}\left(z_{1}\right), z_{2}, \ldots, z_{m}\right)$ such that:

(1) the two complex structures coincide on $\bar{B}$, while

(2) the two complex structures cannot possibly coincide on any neighborhood in $U$ of the point $e_{1} \in \partial B$. 
This means that the standard complex structure on $\bar{B}$ can be extended in inequivalent ways to the border $U \backslash \bar{B}$.

Let $\alpha(z)$ denote the branch of $\sqrt{1-z}$ on $\mathbb{C} \backslash[1, \infty)$ which has positive real part. On the closure $\bar{D}$ we define

$$
\phi(z)= \begin{cases}A z+\exp \left(-\frac{1}{\alpha(z)}\right), & z \neq 1, \\ A, & z=1 .\end{cases}
$$

For every $A \in \mathbb{C}$ this defines a $\mathcal{C}^{\infty}$ function on $\bar{D}$, in the sense of Whitney. For $|A|$ sufficiently large, it defines a biholomorphism of $D$ onto an open domain $G_{\sim}$ in $\mathbb{C}$. By Whitney's theorem, for large $A, \phi$ extends to a smooth diffeomorphism $\widetilde{\phi}$ of an open neighborhood $\omega$ of $\bar{D}$ in $\mathbb{C}$ onto a neighborhood $\Omega$ of $\bar{G}$ in $\mathbb{C}$.

It follows from what was said above that the two complex structures are equivalent on $\bar{D}$, and hence on $\bar{B}$, yielding (1). It remains to establish (2): Consider the function $f\left(z_{1}, \ldots, z_{m}\right)=\widetilde{\phi}\left(z_{1}\right)$ defined on $U$. Then $\left.f\right|_{B}$ is holomorphic with respect to either of the two complex structures, and it is holomorphically extendable across $e_{1}$ with respect to the second one, since it is one of the holomorphic coordinate functions. But $\left.f\right|_{B}$ is not holomorphically extendable across $e_{1}$ with respect to the standard complex structure, because if it were extendable across $e_{1}$, then $A z-\phi(z)$ would have a nonzero holomorphic extension to a neighborhood of 1 in $\mathbb{C}$, while at the same time being flat at 1 ; this gives a contradiction.

\section{Fundamental system of Stein neighborhoods}

Now we return to the situation where $M$ is compact and $X$ is Stein. Theorem 3.1 supplies us with an open complex manifold $\widetilde{X}$, in which $\bar{X}=X \cup M$ appears as a compact domain with a smooth strictly pseudoconvex boundary.

Theorem 4.1. Assume that the compact contact manifold $M$ is the $\mathcal{C}^{\infty}$ intrinsic boundary of a Stein manifold $X$. Then $\bar{X}$ has a fundamental system of open Stein neighborhoods $\{Y\}$ with $\bar{X} \Subset Y \Subset \widetilde{X}$, for each $Y$.

Proof. This now follows from an old result that is proved using the bumping technique of [AG], applied to the strictly pseudoconvex domain $\bar{X}$ in $\widetilde{X}$ : by employing a finite number of small smooth bumps, one can construct an arbitrarily small open neighborhood $Y$ of $\bar{X}$, such that $\partial Y$ is smooth and remains strictly pseudoconvex. Then using local vanishing theorems for coherent analytic sheaves, and the Mayer-Vietoris sequence, applied a finite number of times, it can be shown that the restriction homomorphism

$$
r: H^{q}(Y, \mathcal{F}) \rightarrow H^{q}\left(X,\left.\mathcal{F}\right|_{X}\right)
$$

is an isomorphism for $q>0$, and any coherent analytic sheaf $\mathcal{F}$ on $Y$. We have that $H^{q}(Y, \mathcal{F}) \simeq H^{q}\left(X,\left.\mathcal{F}\right|_{X}\right)=0$ because $X$ is Stein. For more details, see Theorem 5 in AH2], or consult AG, Propositions 16, 17, 21, 22].

\section{Geometric realization of Stein fillable contact structures}

Let $n=1$, so $\operatorname{dim}_{\mathbb{R}} M=3$ and $\operatorname{dim}_{\mathbb{C}} X=2$.

Theorem 5.1. Assume that the 3-dimensional compact contact manifold $M$ is the $\mathcal{C}^{\infty}$ intrinsic boundary of a Stein manifold $X$. Then $M$ has a smooth $C R$ embedding as a closed $C R$ submanifold of $\mathbb{C}^{4}$ (or a closed $C R$ immersion in $\mathbb{C}^{3}$ ). 
Note that this means that the $C R$ structure induced on $M$ from the embedding is the same as the one $M$ inherits from being the boundary of $X$. In particular: the contact structure on $M$ is achieved, via the embedding, by a complex tangent line at each point.

Proof. Choose one of the Stein manifolds $Y \ni \bar{X}$. According to the embedding theorem for Stein manifolds (see [Bi], [Na]), $Y$ has a proper holomorphic embedding as a closed complex submanifold of $\mathbb{C}^{5}$. The restriction of this embedding to $M$ gives a $C R$ embedding of $M$ into $\mathbb{C}^{5} \subset \mathbb{C P}^{5}$. With $N=5$ consider

$$
M^{\prime}=\left\{(p, r) \in M \times \mathbb{C P}^{N} \mid \overline{p r} \text { is tangent to } M \text { at } p\right\} .
$$

Then $M^{\prime}$ is a smooth submanifold of $M \times \mathbb{C P}^{N}$ of real dimension 6 , and $M^{\prime} \ni$ $(p, r) \rightarrow r \in \mathbb{C P}^{N}$ is a smooth map. By Sard's theorem its image has measure zero in $\mathbb{C P}^{N}$, since $2 N>6$. By choosing a point $R_{0} \notin$ its range $\cup M$, and taking a holomorphic projection from $R_{0}$ to a hyperplane $\Sigma$ not containing $R_{0}$, we obtain a $C R$ closed immersion of $M$ into a $\mathbb{C}^{N-1}$.

Next consider

$$
M^{\prime \prime}=\left\{(p, q, r) \mid(p, q) \in M \times M \backslash \Delta, r \in \mathbb{C P}^{N} \text { and } p, q, r \text { are collinear }\right\} .
$$

Then $M^{\prime \prime}$ is a smooth manifold of real dimension 8 , and $M^{\prime \prime} \ni(p, q, r) \rightarrow r \in \mathbb{C P}^{N}$ is a smooth map. Again by Sard's theorem, its image has measure zero, because $2 N>8$. Thus it is possible to choose the point $R_{0}$ so that the $C R$ immersion obtained above is globally one-to-one. As a result we obtain a $C R$ embedding of $M$ into $\mathbb{C}^{4}$. To obtain a $C R$ immersion into $\mathbb{C}^{3}$, we repeat the above projection argument with $N=4$, since then we still have $2 N>6$.

Remark 5.1. When $n=2,3, \ldots$, so that $\operatorname{dim}_{\mathbb{R}} M \geq 5$, the result analogous to Theorem 4.1 holds without any assumption of Stein fillability; one needs only the existence of a $C R$ structure on $M$ which is compatible with the contact structure: assume the $(2 n+1)$-dimensional compact orientable contact manifold $M$ has a smooth $C R$ structure of type $(n, 1)$ which induces the given contact structure and is strictly pseudoconvex. By a theorem of Boutet de Monvel $[\mathrm{BM}], M$ has a smooth $C R$ embedding into $\mathbb{C P}^{N}$, for some $N$. Then we can repeat the argument above and obtain that $M$ has a $C R$ embedding into $\mathbb{C}^{2 n+2}$, or a $C R$ immersion into $\mathbb{C}^{2 n+1}$. The contact structure on $M$ is then achieved, via the embedding, by a tangent affine $\mathbb{C}^{n}$ at each point.

For $C R$ manifolds which are not of hypersurface type, see [HN2].

\section{EquiVAlEnCE OF THE INTRINSIC NOTION AND THE WORKING HYPOTHESIS}

We return to the situation of $\S 1$ and $\S 2$.

Theorem 6.1. Assume that the compact contact manifold $M$ is the $\mathcal{C}^{\infty}$ intrinsic boundary of a Stein manifold $X$. Then the working hypotheses $1^{\circ}, 2^{\circ}, 3^{\circ}, 4^{o}$ of $\S 2$ are satisfied.

Proof. Since a Stein manifold $Y$ has an exhaustion by a smooth strictly plurisubharmonic function, we obtain $1^{\circ}$ and $2^{\circ}$ from Theorems 3.1 and 4.1. To demonstrate $3^{\circ}$ we proceed as follows: Fix a Stein neighborhood $Y$ of $\bar{X}$ in $\widetilde{X}$, a strictly plurisubharmonic function $\psi$ on $Y$, and a Hermitian metric on $Y$. Since $X$ is a domain in 
$Y$, there exists a global defining function $\rho \in \mathcal{C}^{\infty}(Y)$ such that:

$$
\bar{X}=\{x \in Y \mid \rho(x) \leq 0\},\left.\quad d \rho\right|_{M} \neq 0
$$

(see [AH1, Proposition 1.1]). Since $M$ is strictly pseudoconvex, the Levi form $\mathcal{L}(\rho)$ is positive definite at each point of $M$; i.e. has $n$ positive eigenvalues. To obtain $(n+1)$ positive eigenvalues for the complex Hessian $i \partial \bar{\partial} \rho$ near $M$, we replace $\rho$ by a modified global defining function

$$
\widetilde{\rho}=\frac{1}{\lambda}\left\{e^{\lambda \rho}-1\right\},
$$

with the constant $\lambda>0$ chosen sufficiently large. It is easy to verify that there is an open neighborhood $U$ of $M$ in $Y$ in which $\widetilde{\rho}$ is strictly plurisubharmonic, and $d \widetilde{\rho} \neq 0$. Next we modify $\widetilde{\rho}$ to make it strictly plurisubharmonic in an open neighborhood $V$ of $\bar{X}$, and establish $3^{\circ}$ : Let $\chi(\rho)$ be a smooth real convex function of the real variable $\rho$, such that $\chi(\rho)=\rho$ for $\rho \geq-\delta$ and $\chi(\rho)=-2 \delta$ for $\rho \leq-3 \delta$, where $\delta>0$ is chosen so small that $\{x \in \bar{X} \mid-3 \delta \leq \widetilde{\rho}(x) \leq 0\} \subset U$. Let $K \subset X$ be a compact set such that $K \supset\{x \in X \mid \widetilde{\rho}(x) \leq-\delta\}$. Choose a nonnegative smooth cutoff function $\mu \in \mathcal{C}_{0}^{\infty}(X)$ such that $\mu=1$ on a neighborhood of $K$. Consider the function:

$$
\phi=\chi(\widetilde{\rho})+\epsilon \mu \psi,
$$

with a small constant $\epsilon>0$. Then $\left.d \phi\right|_{M}=\left.d \widetilde{\phi}\right|_{M}=\left.d \rho\right|_{M} \neq 0$ and $\bar{X}=\{x \epsilon$ $Y \mid \phi(x) \leq 0\}$ for $\epsilon>0$ taken sufficiently small. The function $\chi(\widetilde{\rho})$ is smooth and weakly plurisubharmonic on $V=X \cup U$. The function $\phi$ is strictly plurisubharmonic in $V$ for sufficiently small $\epsilon>0$. This establishes $3^{\circ}$ without destroying $2^{\circ}$.

The function $\psi$ can be chosen at the beginning to be a Morse function on $Y$; see $\mathrm{AF}$. Hence by construction there is an $\eta>0$ such that $\phi$ has no critical points on $\{x \in V \mid-\eta \leq \widetilde{\rho}(x) \leq \eta\}$, and at most only a finite number of nondegenerate critical points for $\{x \in V \mid \widetilde{\rho}(x) \leq-3 \delta\}$. To obtain $4^{\circ}$, we need to eliminate any degenerate critical points of $\phi$ in $\{x \in V \mid-3 \delta<\widetilde{\rho}(x)<-\eta\}$. Let $\nu \in \mathcal{C}_{0}^{\infty}(X)$, $0 \leq \nu(x) \leq 1$, be a smooth cutoff function with $\nu=1$ on the set $\{x \mid \widetilde{\rho}(x) \leq-\eta\}$. By Sard's theorem we can approximate $\phi$, in the $\mathcal{C}^{2}$-norm on any compact subset of $V$, by a smooth function $\widetilde{\phi}$ which has only nondegenerate critical points; hence $\widetilde{\phi}$ remains strictly plurisubharmonic. Set

$$
\phi_{1}=\nu \widetilde{\phi}+(1-\nu) \phi .
$$

Then for $\phi_{1}-\widetilde{\phi}=(1-\nu)(\phi-\widetilde{\phi})$ there is an estimate

$$
\left|\phi_{1}-\widetilde{\phi}\right|_{2} \leq \text { const }|\phi-\widetilde{\phi}|_{2},
$$

where the norms are $\mathcal{C}^{2}$-norms taken over some compact subset $L \Subset V$, with $\bar{X} \subset \stackrel{o}{L}$. So by taking a sufficiently good approximation $\widetilde{\phi}$ to $\phi$, the function $\phi_{1}$ satisfies $1^{\circ}$, $2^{o}, 3^{o}, 4^{o}$; hence the proof is complete.

\section{Cohomology of the Border}

In spite of the fact that the germ of the border $\widetilde{X} \backslash \bar{X}$ is not unique, it turns out that the germ of its Dolbeault cohomology is unique. 
Theorem 7.1. Assume that the compact contact manifold $M$ is the $\mathcal{C}^{\infty}$ intrinsic boundary of a Stein manifold $X$. Then for any choice of the $\widetilde{X}$, in which $\bar{X}$ is a domain, and for any choice of the Stein neighborhood $Y, \bar{X} \subset Y \Subset \widetilde{X}$, and for any $0 \leq p \leq n+1$, we have:

(1) $H^{p, q}(Y \backslash X) \simeq H^{p, q}(M)=0$ for $0<q<n$,

(2) $H^{p, n}(Y \backslash X) \simeq H^{p, n}(M)$,

(3) $H^{p, n+1}(Y \backslash X)=0$,

with

(4) $\operatorname{dim}_{\mathbb{C}} H^{p, n}(Y \backslash X)=\infty$.

Here $H^{p, q}(Y \backslash X)$ denotes the Dolbeault cohomology of smooth $\bar{\partial}$-closed $(p, q)$ forms on $Y \backslash X$ modulo those which are $\bar{\partial}$ exact in $Y \backslash X$. Note that $Y \backslash X=$ $(Y \backslash \bar{X}) \cup M$ has smooth boundary $M$, and we are requiring here that the differential forms be $\mathcal{C}^{\infty}$ up to $M$. $H^{p, q}(M)$ denotes the $\bar{\partial}_{M^{-} \text {-cohomology of tangential }} \bar{\partial}_{M^{-}}$ closed smooth $(p, q)$-forms on $M$, modulo those that are $\bar{\partial}_{M}$-exact on $M$.

The results (1), (2), (3), (4) are direct consequences of [AH1, AH2, see Theorems 5 and 7], or see Theorem 7.2 in [HN3, and [La].

Remark 7.1. When $q=0$ and $0 \leq p \leq n+1$ we have that $H^{p, 0}(Y \backslash X) \simeq H^{p, 0}(Y)$ and $H^{p, 0}(\bar{X}) \simeq H^{p, 0}(M)$; see [AH1].

\section{REFERENCES}

[AF] A.Andreotti, T.Frankel, The Lefschetz theorem on hyperplane sections, Ann. Math. 69 (1959), 713-717. MR0177422 (31:1685)

[AG] A.Andreotti, H.Grauert, Théorème de finitude pour la cohomologie des espaces complexes, Bull. Soc. Math. France 90 (1962), 193-259. MR0150342 (27:343)

[AH1] A.Andreotti, C.D.Hill, E. E. Levi convexity and the Hans Lewy problem. I. Reduction to vanishing theorems, Ann. Scuola Norm. Sup. Pisa 26 (1972), 325-363. MR0460725 (57:718)

[AH2] A.Andreotti, C.D.Hill, E. E. Levi convexity and the Hans Lewy problem. II. Vanishing theorems, Ann. Scuola Norm. Sup.Pisa 26 (1972), 747-806. MR.0477150 (57:16693)

[Bi] E. Bishop, Mappings of partially analytic spaces, Amer. J. Math. 83 (1961), 209-242. MR0123732 (23:A1054)

[BM] L.Boutet de Monvel, Intégration des équations de Cauchy-Riemann induites formelles, Séminaire Goulaouic-Lions-Schwartz 1974-1975; Équations aux derivées partielles linéaires et non linéaires, vol. Exp. No. 9, Centre Math. École Polytech., Paris, 1975, pp. 1-14.

[Ca] D.Catlin, A Newlander-Nirenberg theorem for manifolds with boundary, Michigan Math. J. 35 (1988), 233-240. MR0959270 (89j:32026)

[Ch] S. Cho, Extension of complex structures on weakly pseudoconvex compact complex manifolds with boundary, Math. Z. 211 (1992), 105-120. MR1179783 (94h:32030)

[DA] J. D'Angelo, Finite type conditions and subelliptic estimates, Modern methods in complex analysis (1992), Ann. of Math. Stud., 137, Princeton Univ. Press, Princeton, NJ, 1995, pp. 63-78. MR1369134 (96k:32030)

[El1] Y.M.Eliashberg, Filling by holomorphic discs and its applications, Geometry of low-dimensional manifolds, 2, London Math. Soc. Lecture Note Ser., vol. 151, Cambridge Univ. Press, Cambridge, 1990, pp. 45-47. MR1171908 (93g:53060)

[El2] Y.M.Eliashberg, Topological characterization of Stein manifolds of dimension > 2, Internat. J. Math. 1 (1990), 29-46. MR.1044658 (91k:32012)

[El3] Y.M.Eliashberg, Symplectic topology in the nineties, Differential Geom. Appl. 9 (1998), 59-88. MR 1636301 (2000a:53136)

[Go] R.E.Gompf, Handlebody construction of Stein surfaces, Ann. of Math. 148 (1998), 619693. MR1668563 (2000a:57070)

[He] D. Heunemann, Extension of the complex structure from Stein manifolds with strictly pseudoconvex boundary, Math. Nachr. 128 (1986), 87-101. MR0855943|(87j:32057) 
[H1] C.D.Hill, What is the notion of a complex manifold with a smooth boundary?, Algebraic Analysis I (Kashiwara and Kawai, eds.), Academic Press, Boston, MA, 1988, pp. 185-201. MR0992454 (90e:32009)

[H2] C.D.Hill, A family of exotic CR structures on $S^{3} \times S^{2}$, Analyse complexe multivariable: récents développements (Guadeloupe, 1988) Sem. Conf., 5, EditEl, Rende, 1991, pp. 105110. MR1228874 (94f:32039)

[H3] C.D.Hill, Counterexamples to Newlander-Nirenberg up to the boundary, Several complex variables and complex geometry, Part 3 (Santa Cruz, CA, 1989), Proc. Sympos. Pure Math., 52, Part 3, Amer. Math. Soc.,, Providence, RI, 1991, pp. 191-197. MR1128593 (92m:32027)

[HJ] N.Hanges, H.Jacobowitz, A remark on almost complex structures with boundary, Amer. J. Math. 111 (1989), 53-64. MR0980299 (90m:32038)

[HN1] C.D.Hill, M.Nacinovich, A collar neighborhood theorem for a complex manifold, Rend. Sem. Mat. Univ. Padova 91 (1994), 24-30. MR.1289628 (95g:32015)

[HN2] C.D.Hill, M.Nacinovich, The topology of Stein CR manifolds and the Lefschetz theorem, Ann. Inst. Fourier (Grenoble) 43 (1993), 459-468. MR.1220278 (94d:32012)

[HN3] C.D.Hill, M.Nacinovich, Pseudoconcave $C R$ manifolds, Complex analysis and geometry (Trento, 1993), Lecture Notes in Pure and Appl. Math., 173, Dekker, New York, 1996, pp. 275-297. MR,1365978 (97c:32013)

[La] H.B. Laufer, On the infinite dimensionality of the Dolbeault cohomology groups, Proc. Amer. Math. Soc. 52 (1975), 293-296. MR0379887 (52:792)

[Le1] L. Lempert, On three-dimensional Cauchy-Riemann manifolds, J. AMS 5 (1992), 923-969. MR1157290 (94d:32025)

[Le2] L.Lempert, Embeddings of three-dimensional Cauchy-Riemann manifolds, Math. Ann. 300 (1994), 1-15. MR1289827 (95j:32029)

[Le3] L. Lempert, Algebraic approximations in analytic geometry, Invent. Math. 121 (1995), 335-353. MF 1346210 (97k:32021)

[LiM] P.Lisca, G.Matić, G. Stein 4-manifolds with boundary and contact structures.; Symplectic, contact and low-dimensional topology (Athens, GA, 1996), Topology Appl. 88 (1998), 5566. MR 1634563 (99f:57037)

[Na] R.Narasimhan, Imbedding of holomorphically complete complex spaces, Amer. J. Math. 82 (1960), 917-934. MR0148942 (26:6438)

[Oh] T. Ohsawa, Holomorphic embeddings of compact s.p.c. manifolds into complex manifolds as real hypersurfaces, Differential geometry of submanifolds (Kyoto, 1984), Lecture Notes in Math., 1090, Springer, Berlin, 1984, pp. 64-76. MR0775145 (86j:32047)

Department of Mathematics, SUNy at Stony Brook, Stony Brook, New York 11794

E-mail address: dhill@math.sunysb.edu

Dipartimento di Matematica, Universitì di Roma "Tor Vergata", via della Ricerca Scientifica, 1 - 00133 - Roma, Italy

E-mail address: nacinovi@mat.uniroma2.it 